\title{
STARE: Spatio-Temporal Attention Relocation for Multiple Structured Activities Detection
}

\author{
Kyuhwa Lee*, Dimitri Ognibene, Hyung Jin Chang*, Tae-Kyun Kim, Yiannis Demiris
}

\begin{abstract}
We present a Spatio-Temporal Attention Relocation (STARE) method, an information-theoretic approach for efficient detection of simultaneously occurring structured activities. Given multiple human activities in a scene, our method dynamically focuses on the currently most informative activity. Each activity can be detected without complete observation, as the structure of sequential actions plays an important role on making the system robust to unattended observations. For such systems, the ability to decide 'where' and 'when' to focus is crucial to achieving high detection performances under resource bounded condition. Our main contributions can be summarized as follows: (i) Information-theoretic dynamic attention relocation framework that allows the detection of multiple activities efficiently by exploiting the activity structure information. (ii) A new high-resolution dataset of temporally-structured concurrent activities. Our experiments on applications show that the STARE method performs efficiently while maintaining a reasonable level of accuracy.
\end{abstract}

Index Terms-Activity detection, visual attention, resource allocation, stochastic context-free grammars.

\section{INTRODUCTION}

Active vision [1] and visual attention [2] systems dynamically select parts of a visual input for efficient processing, which have high importance when the amount of visual input produces an excessive computational load. Common techniques used are applying a region of interest (ROI), camera view selection, and changing the pan, tilt, zoom (PTZ) camera parameters. The active vision systems have been gaining more interest in vision community as their performances are comparable or even surpass conventional passive vision systems [2]-[5]. These systems have been applied in a wide range of areas such as surveillance [6], [7], object detection and

Kyuhwa Lee is with the Chair in Brain-Machine Interface lab, Center for Neuroprosthetics, School of Engineering, École Polytechnique Fédérale de Lausanne (EPFL), Switzerland.

Dimitri Ognibene is Marie Skłodowska-Curie COFUND Fellow at DTIC, Universitat Pompeu Fabra, Spain.

Hyung Jin Chang and Yiannis Demiris are with the Personal Robotics Lab, Department of Electrical and Electronic Engineering, Imperial College London, London, UK.

Tae-Kyun Kim is with the Computer Vision and Learning lab, Department of Electrical and Electronic Engineering, Imperial College London, London, UK.

* Corresponding authors. tracking [8]-[10], object recognition [4], [5], [11], camera view selection [6] and action recognition [12]-[15].

One of the main purposes of these methods is to discard in a priori a part of the information using one or more attention policies. This additional layer of complexity can be, however, non-trivial to deal with if a system has to process complex dynamic scenarios. Thus, active vision systems have been mostly applied on the recognition of isolated and temporally unstructured actions [14]. We are instead interested in scenarios where several independent, long structured activities can occur in parallel. To deal with these scenarios, we develop a dynamic attention relocation method which makes use of the information acquired during the activity detection process, aiming to detect such activities with less computational resources while maintaining comparable detection performances.

State-of-the-art complex structured activity analysis techniques [16] often utilize syntactic approaches [17], [18]. We employ stochastic context-free grammars (SCFG) [19], [20] to both represent and detect human activities. SCFG-based methods have been primarily used as activity recognizer instead of generator (e.g. [19], [21]-[24]), which is not sufficient for our purpose. Hence, we augment the conventional parser by exploiting the structural information encoded in the SCFG to predict the next successive actions while recognizing, which will provide crucial information to the attention relocation process.

In this paper, we present a spatio-temporal attention relocation (STARE) method for efficient activity detection using the knowledge about long-term structured activities. Although our system does not necessarily aim to work in real time, we aim to detect multiple activities efficiently with reasonable accuracy while not committing a full observation. This is done by exploiting the structural constraints of actions. An overview of our system is illustrated in Figure 1 .

Our approach is implemented in three layers: The low layer extracts visual features from the attended area, while the middle layer recognizes elementary human actions. The high layer predicts the next successive actions given the current and previous action observations. These predicted actions play a vital role for computing 


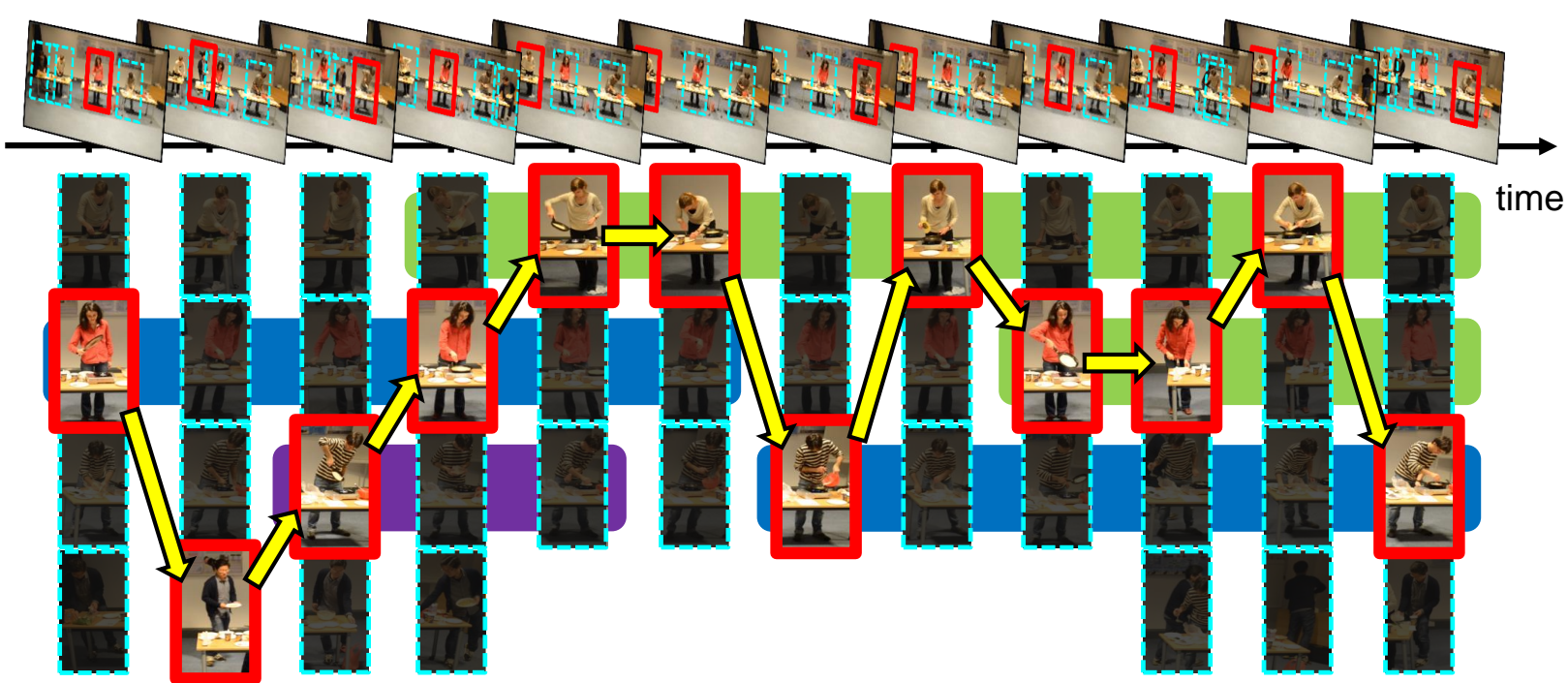

Fig. 1: Illustrative explanation of attention relocation by STARE method. The red boxes indicate where the system made an observation at each sampling time to dynamically focus on the action that is expected to be the most informative. The color bars on the background denote different activity classes. (Best shown in color.)

the following information-theoretic attention relocation policies: a) Allocate resources on an area with the highest activity detection confidence. b) Allocate more resources on an area that is most likely to contain an undetected activity. c) The combination of these two policies for balanced approach. The main contributions of our work can be summarized as follows:

1) Information-theoretic active attention relocation: We present an attention relocation system suitable for detecting concurrent activities under bounded computational resources by exploiting activity structures. This is done through an SCFG-based action prediction, which can predict the successive actions given the previous observation actions by exploiting temporally structured activity information. The predicted future actions provide crucial information to our attention relocation system. This method differs from the traditional use of SCFG parsing, e.g. [19], [21], [24]-[27], where the parser is used as a recognizer (classifiers). We use the parser as a recognizer as well as generator to predict the next possible actions and determine where to allocate resources in the next time step.

2) New dataset of temporally structured concurrent activities: We present a new activity dataset containing 6 different structured cooking activities with distractors where multiple activities may occur concurrently. To the best of our knowledge, this is the first long-term structured human activity dataset with multiple classes in full-HD resolution (1080p).

Last but not least, it is also worth mentioning that the principles of our model are inspired from the neuroscience field, such as the hierarchical representation of actions ([25], [28]-[30]), the internal simulation of future actions ([28]) and attention control ([31]-[36]).

\section{RELATED WORKS}

Attention relocation (control) systems have been widely used to reduce the computational cost of visual processing by focusing on more informative parts. As explained in several recent reviews [2], [37], the current studies focus mainly on approaches which are based on spatially and temporally localized visual signals, which are not sufficient to model the information requirements of complex tasks in a dynamic context, such as activity detection. As admitted in [2], such approaches put more emphasis on predicting human fixations using local and unstructured temporal information. To elaborate the differences, we list some representative works in three major categories related to our work.

1) Bottom-up attention relocation systems: Bottomup attention controllers, both bio-inspired approach [38] and information-theoretic approach [39], make decisions based on low-level visual features. Jiang et al. [8] recently presented a visual-cue-based attentional region detection in a static image, where [40] combines an outdoor scene classification system based on 'gist', computed from multi-scale set of early-visual features, with a saliency based attention mechanism. Denzler et al. [41] studied dynamic attention selection for object tracking using the uncertainty information acquired from Kalman filter. Chang et al. [42] presented a dynamic bottom-up attention control scheme for speeding up the background subtraction process. They generated a dynamic attention probability map by considering specifically designed at- 
tention properties of the application. Since these bottomup systems do not consider high-level structures, the information they select does not necessarily contribute to improve high-level tasks such as activity detection and recognition.

2) Top-down attention relocation systems: Navalpakkam et al. [43] augment the bio-inspired model [38] by adding a top-down component of visual attention, which computes the gist of a scene to acquire the prior distribution of a given object and select taskrelated features to optimize static object detection. Vijayanarasimhan and Kapoor [9] tackle an object detection problem which uses an approximated value of information to prioritize more informative features among the pool of features such as local descriptors, textures and color histograms. The above mentioned methods depend on static features, which is not optimal in dynamic scenarios.

For dynamic top-down attention relocation, Sommerlade et al. [6] use mutual information maximization technique to detect and track multiple targets based on their motion, whereas our method is based on type of the action being performed. Although [12] represents human actions using layered hidden Markov models, they model only unstructured actions such as phone conversation or face-to-face conversation, whereas our approach considers the detection of concurrently occurring temporally structured activities. Ognibene et al. [14] model a set of actions as a mixture of Kalman Filters and compute the maximum information gain to select the view of the camera between the human's hand and the expected positions of action target candidates. However, this method differs from our approach since they rely only on the object position and considers only a single, unstructured action at time.

Finally, although there have been also efforts [44], [45] to detect actions as early as possible without watching in full, their models are more suited for detecting actions rather than activities and cannot be used to predict future actions which are crucial for our purpose.

3) Comparison of our dataset with previous datasets: Existing benchmark datasets are not suitable for our evaluation. Our requirements are: (1) multiple human objects in a scene with independent activities occurring in parallel; (2) multiple samples per activity class; (3) temporally structured and long activities (at least 60 seconds); (4) high resolution. We compare our new dataset with the previous datasets using the above criteria in Table I] A detailed description about the new dataset will be given in Section IV-C

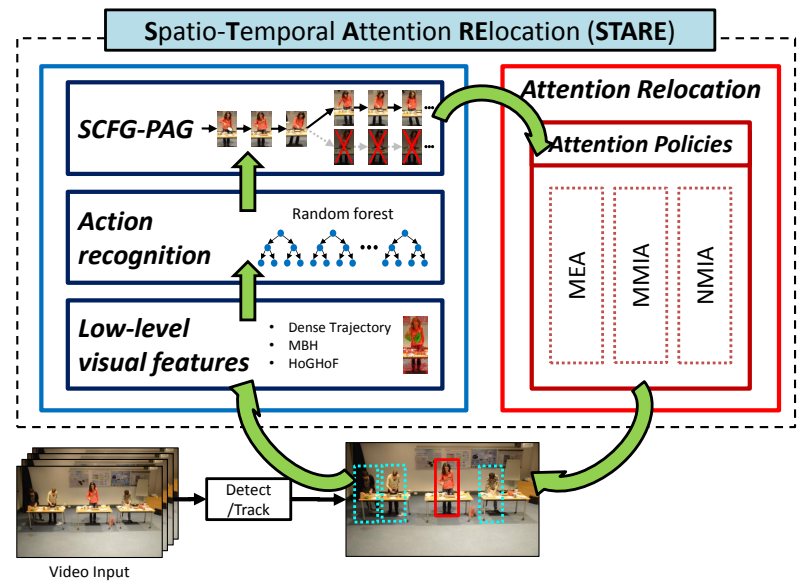

Fig. 2: Overview of the proposed STARE system

\section{iII. Spatio-Temporal attention Relocation (STARE) FOR EFFICIENT ACTIVITY DETECTION}

Our goal is to detect ongoing activities in scenarios where several different activities may evolve simultaneously over time with different speeds while attending (watching) only one of the candidate areas at a time. The base idea of our approach is that past observed actions and the temporal structure of activities are valuable sources of information not only for detecting ongoing activities but also for deciding where to attend among candidate areas in the next time step.

We adopt a probabilistic generative model, Stochastic Context-Free Grammars (SCFG), to represent the temporal structure of activities and encode the observed actions history. By efficiently interleaving the action (observations) parsing and action prediction, in parallel for each activity we can predict the next expected action observation distribution using the activity structure and past observations. Using this expected action observation distributions we estimate the potential improvement of the activity recognition achievable corresponding to the different candidate areas to attend.

We implement and compare three informationtheoretic attention policies, each of which having distinctive properties suitable for different situations. Intuitively, they are: 1) always prefer to watch an area with the highest activity detection confidence, 2) watch less on highly predictable areas and prefer to focus more on the areas which are likely to provide a higher amount of new information, and 3) the combination of these two policies for balanced approach. The attention policy formulations can be found in Section III-C Figure 2 shows an overview of the proposed STARE system.

\section{A. Visual Feature Extraction and Action Detection}

The lower layer of our system computes visual features only for the selected window regions of an input 
TABLE I: Comparison with previous datasets

\begin{tabular}{|c|c|c|c|}
\hline Dataset & Resolution & Example Categories (not exhaustive) & Comments \\
\hline BEHAVE & $640 \times 480$ & Walk together, meet, chase & Multiple people but no structured activity. \\
\hline $\begin{array}{l}\text { UCR } \\
\text { Videoweb }\end{array}$ & $640 \times 480$ & Talk on phone, push button, wave hand, walk backwards & $\begin{array}{l}\text { Multiple long-sequence videos and multiple people, but no structured } \\
\text { activities. }\end{array}$ \\
\hline CMU Kitchen & $1024 \times 768$ & Cooking: brownie, egg, pizza, salad, sandwich & single person per sample. \\
\hline CMU Mocap & $352 \times 240$ & $\begin{array}{l}1 \text { person: martial arts, basketball, acrobatics } \\
2 \text { people: quarrel, pull-resist, conversation }\end{array}$ & $\begin{array}{l}\text { Up to } 2 \text { people for unstructured short-term activities, and } 1 \text { person with } \\
\text { actions only (no structured activities). }\end{array}$ \\
\hline MuHAVi & $720 \times 576$ & Crawl on knees, climb a ladder, pick up and throw an object & Multiple views but single person per sample. Short-term activities only. \\
\hline ViSOR & $800 \times 600$ & Drinking, leaving an object, sitting on a chair, running & multiple people but no structured activity. \\
\hline Weizmann & $180 \times 144$ & Jumping jack, gallop sideways, swing a bag, two-hand wave & No structured activities, low resolution, single p \\
\hline CAVIAR & $384 \times 288$ & $\begin{array}{l}\text { Set1: Walking (straight, return, B-line), browsing a shop } \\
\text { Set2: People entering and exiting a store }\end{array}$ & $\begin{array}{l}\text { Low resolution, short-term activities only. Not enough samples due to a } \\
\text { large number of classes. (less than } 10 \text { samples per class) }\end{array}$ \\
\hline CANDELA & $352 \times 288$ & $\begin{array}{l}\text { Pick up object, sit and leave bag, sit and handover a bag, } \\
\text { three people gather and depart, park car and walk away }\end{array}$ & Low resolution, no concurrent activities. \\
\hline UT-Tower & $360 \times 240$ & Digging, carrying, jumping, Walking & Low resolution, short-term activities only. \\
\hline UT-Interaction & $720 \times 480$ & Shake-hands, point, hug, push, kick, Punch & No structured activities, single person. \\
\hline KTH & $160 \times 120$ & Walking, jogging, boxing, hand clapping & No structured activities, low resolution, single person. \\
\hline TRECVID & $720 \times 576$ & Running, meeting, embracing, pointing, opposing flow & No structured activities. \\
\hline VIRAT & $1920 \times 1080$ & $\begin{array}{l}\text { Person loading/unloading an object to vehicle, person open- } \\
\text { ing/closing a trunk, person getting into/out of a vehicle. }\end{array}$ & $\begin{array}{l}2 \text { types of long-term structured activities (delivery/take away) exist in less } \\
\text { than } 20 \text { video samples. Only few scenarios have concurrent activities. }\end{array}$ \\
\hline
\end{tabular}

video using dense trajectory features [46], motion boundary histogram [47], and histogram of optical flow and oriented gradients [48]. A supervised visual codebook (check also [49]) is learned from these visual features using an extremely clustered random forest [50]. The middle layer of the system computes a histogram of codewords obtained from sliding windows over time and classify actions with random forest [51]. For other approaches applicable at this stage see [49], [52]. At this step, we obtain a sequence of actions with their likelihoods.

\section{B. Probabilistic Parsing of Activities}

The higher layer of the system performs activity detection and predictive action generation by making use of the likelihoods computed by the action classifiers in the middle layer. The input is the distribution of action likelihoods, which is passed to the SCFG parser in a similar fashion as in [19]. We compute the next predicted action likelihoods after parsing each input symbol. This generated action prediction information is used in Section III-C. It is worth noting that we use a robust version of SCFG presented presented in [19] which takes into account the likelihoods of each input symbol, i.e. action likelihoods, in addition to the standard rule probabilities.

In this paper, we use the same notations of the SCFG framework used in [19] for consistency. An activity is represented as an SCFG composed of 4 conventional components, i.e. $G=\{R, T, N, S\}$ : Production rules $(R)$, Terminal symbols $(T)$, Non-terminal symbols $(N)$ and the Start symbol $(S)$, a special non-terminal symbol. The production rules $R$ are similar to the ones used in standard context-free grammars except that every rule is specified with a rule probability. Similarly, terminal symbols $T$ are specified with probability values corresponding to action symbols in our case. The nonterminals $N$ correspond to the abstraction of symbols.
A production rule states how a non-terminal symbol $X$ should generate a set of symbols, which takes the form $X \rightarrow \lambda$, where $\lambda \in(N \cup T)^{*}$ and $P(X \rightarrow \lambda)$ states the probability of $X$ generating $\lambda$. The order of the symbols in the right-hand side of the production rule enforces the actions to be occurred in the same order. The input is a time series of $n$-dimensional vectors where the $n$-th element of a vector contains the likelihood of the $n$-th action class produced by the corresponding action detector (sec. III-A).

A state of the parser is expressed as:

$$
i: X \rightarrow \lambda . Y \mu
$$

where $X \rightarrow \lambda Y \mu$ is a production rule defined in the grammar. '? marker is the parser's current reading position in the rule and $i$ is the position in the input stream, i.e. $i$-th observation. $X$ and $Y$ denote nonterminal symbols while $\lambda$ denotes the parsed symbols in the context of $X$ and $\mu$ denotes the expected terminal symbols as the next input.

For each input, the parser iteratively executes the three steps scanning step, completion step and prediction step to build the parse tree. 1) Scanning step reads a symbol from the input stream and matches it with the initial set of rules, generating a new set of states by increasing $i$ index in the state definition (Eq. 1). 2) completion step updates the marker positions in all pending derivations. 3) prediction step hypothesizes the possible continuation of the input symbols. Please refer to [19], [53] for more detailed explanation of each step as it is outside of the scope of this paper. Here, we explain the prediction step since it provides the information we use to predictively control attention. In the prediction step, the parser computes the next expected input symbols to the system:

$$
\left\{\begin{array}{l}
i: X_{k} \rightarrow \lambda . Y \mu[\alpha, \gamma] \\
Y \rightarrow \mu
\end{array} \quad \Rightarrow \quad i: Y_{i} \rightarrow . \nu\left[\alpha^{\prime}, \gamma^{\prime}\right]\right.
$$




$$
\begin{aligned}
\alpha^{\prime} & =\sum_{\forall \lambda, \mu} \alpha\left(i: X_{k} \rightarrow \lambda . Y \mu\right) P(Y \rightarrow \nu), \\
\gamma^{\prime} & =P(Y \rightarrow \nu)
\end{aligned}
$$

where $\Rightarrow$ denotes a transition between parser states when the grammar rule $Y \rightarrow \mu$ is applied. $\alpha$ is a forward probability that represents the probability of the parsed terminal symbols until the $i$-th index of the input stream, whereas $\gamma$ is the inner probability, which represents the probability of a substring that starts at input index $k$ and ends at $i . \nu$ denotes the possible continuation of input symbols at the current parsing step, i.e. the expected observation in the next time step.

Let $j$ be a state of the parser, $\nu_{0}^{j}$ be the first possible continuation symbol of $\nu^{j}$ in Eq. 2 and $\alpha_{i}(j)$ a forward probability of the hypothesis at the $i$-th index. Let $o_{t}$ be an observation at time $t$. We can compute the most probable likelihood vector $o_{t+1}$ given the past observations $o_{1, \ldots, t}$ and the grammar structure. For each symbol $s \in T$ the corresponding likelihood $o_{t+1}(s)$ can be computed by searching for the state $j$ with the highest forward probability in $\mathcal{J}$ among those where $s$ corresponds to $\nu_{0}^{j}$ :

$$
o_{t+1}(s)=P\left(s \mid o_{1, \ldots, t}\right)=\left.\frac{1}{\eta} \arg \max _{j \in \mathcal{J}}\left(\alpha_{t}^{\prime}(j)\right)\right|_{\nu_{0}^{j}=s} .
$$

Here, $\eta$ is the normalization factor so that $\sum_{s \in T} P\left(s \mid o_{1, \ldots, t}\right)=1$. This is an approximation since the parse trees with very low likelihood are pruned instead of being expanded exhaustively to increase the parsing speed, as also done in [19], [54], [55]. $o_{t+1}$ will be used to characterize the expected observation distribution for a given grammar.

\section{Information-theoretic Attention Relocation}

Computation of Information Scores: Let $\mathcal{A}^{w}$ be our stochastic variable that describes the activity taking place in a candidate area (window) $w \cdot \mathcal{A}^{w}$ can be one of $L$ activities, $A_{1}, A_{2}, \ldots, A_{L}$. Each kind of activity $A_{l} \in L$ corresponds to a different grammar. An observation $o_{t}^{w}$ is the likelihood distribution of actions after making an observation, provided by the middle layer (Sec. IIII-A), at time $t$. For simplicity, $w$ will be omitted when there is no ambiguity and we will denote observations up to time $t, o_{1, \ldots, t}$, as simply $\mathbf{o}_{t}$. Let $\hat{o}_{t+1}$ be a random variable that denotes the predicted future observation in the next time step and $H\left(\mathcal{A} \mid \mathbf{o}_{t}\right)$ the entropy of current activity given past observations, i.e.

$$
H\left(\mathcal{A} \mid \mathbf{o}_{t}\right)=-\sum_{A \in \mathcal{A}} P\left(A \mid \mathbf{o}_{t}\right) \log P\left(A \mid \mathbf{o}_{t}\right)
$$

where $P\left(A \mid \mathbf{o}_{t}\right)$ term can be obtained from the SCFG parser of grammar $A$ after finishing the completion step by choosing the maximum forward probability computed so far. Then the mutual information between the current activity and the expected observation at time $t+1$ given the past observations is:

$$
I\left(\mathcal{A} ; \hat{o}_{t+1} \mid \mathbf{o}_{t}\right)=H\left(\mathcal{A} \mid \mathbf{o}_{t}\right)-H\left(\mathcal{A} \mid \hat{o}_{t+1}, \mathbf{o}_{t}\right) .
$$

which tells us how much the uncertainty of activities will change if we make an observation in the next time step.

In equation 3, we assumed we have only a single grammar. Since we have multiple grammars, one parser running for each grammar, we re-write the equation 3 as:

$$
\hat{o}_{t+1}^{A}(s)=P\left(s \mid \mathbf{o}_{t}, A\right)=\left.\frac{1}{\eta} \arg \max _{j \in \mathcal{J}}\left(\alpha_{t}^{\prime}(j)\right)\right|_{\nu_{0}^{j}=s} ^{\mathcal{A}=A} .
$$

The computation of $H\left(\mathcal{A} \mid \hat{o}_{t+1}, \mathbf{o}_{t}\right)$ requires $P\left(\mathcal{A} \mid \hat{o}_{t+1}, \mathbf{o}_{t}\right)$, which can be obtained by exploiting the internal parser states according to Eq. 6 For each activity grammar $A$, we obtain different predicted observations $\hat{o}_{t+1}^{A}$, i.e. a vector of symbol likelihoods. For each of these predicted observations, we advance each parser corresponding to a different grammar by feeding back $\hat{o}_{t+1}^{A}$ to obtain $P\left(\mathcal{A}=A \mid \hat{o}_{t+1}^{A}, \mathbf{o}_{t}\right)$. After computing $P\left(\mathcal{A}=A \mid \hat{o}_{t+1}^{A}, \mathbf{o}_{t}\right)$, we roll back the parser's state to the previous state before feeding $\hat{o}_{t+1}^{A}$ to get ready for the next input.

At time $t$ an observation is made only on the attended object $w_{t} . \mathcal{A}^{w}$ will be updated by advancing the parser with the observation received at every time step. Let's denote an attended object at time $\mathrm{t}$ and $\mathrm{t}+1$ as $\tilde{w}_{t}$ and $\tilde{w}_{t+1}$ respectively. For all other objects that are not going to be attended $\left(w \neq \tilde{w}_{t+1}\right)$, observations are given as a uniform distribution over the features since there is no new information. This "dummy" observation can be understood as a "missing" data from the parser's point of view. Let $W_{N}=\left\{w_{1}, w_{2} \ldots\right\}$ be a set of objects that were not attended and $W_{A}$ be a set of attended objects $\left(W=W_{A} \bigcup W_{N}\right)$. An object $w$ that was not observed maintain the same activity distribution of the previous time step:

$$
H\left(\mathcal{A}^{w} \mid \hat{o}_{t+1}^{w}, \mathbf{o}_{t}^{w}\right)=H\left(\mathcal{A}^{w} \mid \mathbf{o}_{t}^{w}\right) \quad \forall w \in W_{N} .
$$

We now discuss three policies for selecting which object $w$ to attend in the next time step.

1) Minimum Entropy Attention(MEA) Policy: A straightforward object selection policy could be to always select the object with the minimum expected entropy at time $t+1$ :

$$
\tilde{w}_{t+1}^{M E A}=\arg \min _{w \in W} H\left(\mathcal{A}^{w} \mid \hat{o}_{t+1}^{w}, \mathbf{o}_{t}^{w}, \tilde{w}_{t+1}=w\right) .
$$

This approach drives the system to always relocate attention by following an object that is most likely to have a known activity. Once the current object activity turns out to be reliable, the system will keep focusing on 
the current object. However, in scenarios where there are more than one object performing an activity, the system will fail to detect multiple activities.

2) Maximum Mutual Information Attention(MMIA) Policy: Instead of following an object with the minimum entropy, we try to minimize the overall expected entropy across all existing object in the scene. Thus, for each candidate attended object $w \in W$ at time $t$, we define a attention score $\mathcal{S}^{w}$ as a form of summation of entropies:

$$
\begin{aligned}
\mathcal{S}^{w} & =\sum_{v \in W} H\left(\mathcal{A}^{v} \mid \hat{o}_{t+1}^{v}, \mathbf{o}_{t}^{v}, \tilde{w}_{t}=w\right) \\
& =H\left(\mathcal{A}^{w} \mid \hat{o}_{t+1}^{w}, \mathbf{o}_{t}^{w}, \tilde{w}_{t+1}=w\right)+\sum_{v \in W_{N}} H\left(\mathcal{A}^{v} \mid \mathbf{o}_{t}^{v}\right) .
\end{aligned}
$$

The optimal attention relocation policy after making an observation at time $t$ is thus:

$$
\tilde{w}_{t+1}^{M M I A}=\arg \min _{w \in W} \mathcal{S}^{w} .
$$

Consider now two different object selection cases for the next time step. One is selecting $w_{j}$ and the other is selecting $w_{k}$, with $j \neq k$. Using Eq. 7 and Eq. 5, the attention score difference between the two selections can be derived as follows:

$$
\begin{aligned}
\mathcal{S}^{w_{j}}-\mathcal{S}^{w_{k}}= & \\
= & \left(H\left(\mathcal{A}^{w_{j}} \mid \hat{o}_{t+1}^{w_{j}}, \mathbf{o}_{t}^{w_{j}}, \tilde{w}_{t+1}=w_{j}\right)+H\left(\mathcal{A}^{w_{k}} \mid \mathbf{o}_{t}^{w_{k}}\right)\right) \\
& -\left(H\left(\mathcal{A}^{w_{j}} \mid \mathbf{o}_{t}^{w_{j}}\right)+H\left(\mathcal{A}^{w_{k}} \mid \hat{o}_{t+1}^{w_{k}}, \mathbf{o}_{t}^{w_{k}}, \tilde{w}_{t+1}=w_{k}\right)\right) \\
= & I\left(\mathcal{A}^{w_{k}} ; \hat{o}_{t+1}^{w_{k}} \mid \mathbf{o}_{t}^{w_{k}}\right)-I\left(\mathcal{A}^{w_{j}} ; \hat{o}_{t+1}^{w_{j}} \mid \mathbf{o}_{t}^{w_{j}}\right) .
\end{aligned}
$$

Thus, selecting an object $w$ which makes the attention score minimum is equivalent to selecting $w$ of the maximum mutual information between $\mathcal{A}^{w}$ and $\hat{o}_{t+1}^{w}$ :

$$
\tilde{w}_{t+1}^{M M I A}=\arg \min _{w \in W} \mathcal{S}^{w}=\arg \max _{w} I\left(\mathcal{A}^{w} ; \hat{o}_{t+1}^{w} \mid \mathbf{o}_{t}^{w}\right) .
$$

3) Normalized Mutual Information Attention(NMIA) Policy: The previous two policies have distinctive contrasting behaviors. The MEA policy has a strong tendency towards an object with the lowest uncertainty, which can lead to higher accuracy but poor at detecting multiple activities occurring in parallel. The MMIA policy prefers objects that are less predictable. This is good for multiple activity detection while minimizing the accuracy loss. However, it may prefer an object that does not perform any activity, i.e. distractors, compared to other objects. Hence, we propose the third policy which aims to balance between the previous two. We adopt the normalized mutual information suggested in [56], [57], which has the effect of reducing the tendency of getting stuck in the lowest uncertainty window as well as the effect of the distractors.

$\tilde{w}_{t+1}^{N M I A}=\arg \max _{w}\left(\frac{I\left(\mathcal{A}^{w} ; \hat{o}_{t+1}^{w} \mid \mathbf{o}_{t}^{w}\right)}{\max \left(H\left(\mathcal{A}^{w} \mid \mathbf{o}_{t}^{w}\right), H\left(\hat{o}_{t+1}^{w} \mid \mathbf{o}_{t}^{w}\right)\right)}\right)$

\section{Evaluation AND Analysis}

In our evaluation, we focus on how the proposed STARE system performs on multiple activity tracks. The goal of the system is to detect as many activities as possible in various conditions. We assume that we use electronic Pan-Tilt-Zoom (ePTZ) cameras, where human object detection is already performed, as we are interested in the selection of human object using our activity-level attention system. For comparison, we conduct our experiments using classical scheduling policies such as random and round-robin, a bottom-up saliency model proposed by Itti et al. [38], and active attentional framework proposed by Chang et al. [42]. For [38], we obtain a saliency map using their method and add up the saliency values within each human bounding box, and select the human object with the maximum value. For [42], we apply the action likelihood value to be used as the detection mask score in their method. We adapt their sampling policy only, without their low-level and mid-level processing steps, to compare the performance of different attention policies.

\section{A. Implementation Details}

1) Visual codebook: We use the low-level visual feature extraction methods reported in [46] to compute the dense trajectories of points over a fixed temporal length (15 frames) and the motion boundary histograms [47] and histograms of optical flow and oriented gradients [48] around the tracked points (32 neighborhood pixels, 2 spatial cells, 3 temporal cells). From the extracted descriptors in the training set, we compute a supervised 5000-dimensional visual codebook using an extremely clustered random forest [50] (5 trees, 1000 leaf nodes per tree) from 5000 samples per class.

2) Action recognition: The histograms are computed using the codebook over a fixed temporal window size (60 frames) for training classifiers. We train a random forest classifier implemented in [51] (50 trees, 50 maximum depth) over the training histograms. The output of the classifier is a likelihood distribution of actions, which is fed into our high-level activity detector.

3) SCFG parsing and action prediction: We use the SCFG parsing and action prediction method explained in Section III-B to compute the expected change in action and activity likelihoods. To speed up the SCFG parsing process, we prune parse trees having very low likelihoods. The structure of the activities are given in grammars which directly reflects the defined activity specifications, and the rule parameters for each grammar are computed from the relative occurrences of action symbol in each activity from the training set.

Probabilistic grammars have often been effectively used by manually defining simple grammars using prior 


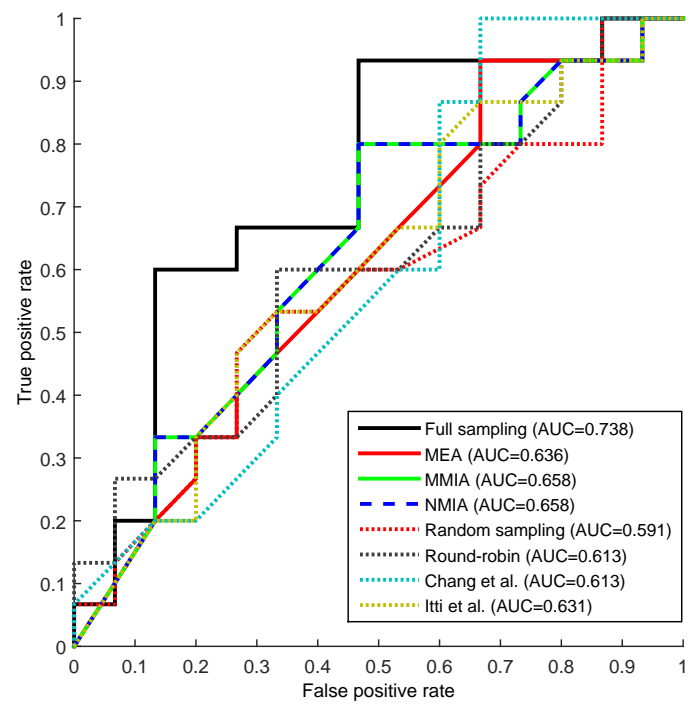

Fig. 3: VIRAT dataset. ROC curves obtained under different attention policies and respective area values. Policies MMIA and NMIA exhibit the same performance in this graph. Best viewed in color.

task knowledge without excessive parameter tuning [19], [21]. We followed a similar approach in this work and defined the grammars in a straightforward manner to measure the robustness of the system with noisy input. Note, however, that activity grammars can be also learned from data [23], [58]-[60] when enough data are available. We have previously shown that SCFG can be constructed from data with limited hand labeling [20], [26].

\section{B. VIRAT Dataset}

We test the STARE framework using a high-resolution VIRAT dataset[61]. We use the videos from "VIRAT_S_000001.mp4" to "VIRAT_S_000102.mp4" since they contain long-term temporally structured activities of two interesting scenarios: Collection and Delivery. There are 16 activities, 76 actions (excluding wandering), and 152 human objects in total. It comes with annotation which includes: Load/Unload an object (2 actions), Open/Close a car trunk (2 actions), Get in/out of a car ( 2 actions). In addition to these actions, we denote all standing/wandering movements between any two actions as "wander" action. Since the annotation is provided only at the action level, we define the temporal range activities that contain the sequence of these actions. At the end of every activity, Viterbi parsing is performed to compute the activity likelihoods, normalized by the number of observations. We show ROC curves in Fig. 3

The Delivery activity is defined as: Get out of a car, Open a trunk, Unload an object, Close the trunk and Get in to the car, whereas Collection activity is the same as Delivery except it has load instead of unload. The temporal lengths of these activities allow enough time for our attention system to show effect on our visual system over long time period. It is important to note that in this dataset, an activity may not be carried out by a single person, e.g. one person unloading while another closing the trunk, with abundance of occlusions, which makes the dataset quite challenging.

\section{Crêpe Dataset}

The set-up we choose in this new datase ${ }^{1}$ is similar to that of a restaurant kitchen scenario, where several chefs and waiters/waitresses are preparing dishes for customers. The chefs are target objects and waiters/waitresses are distractors. The chefs only cook, while waiters and waitresses enter and exit the scene at any time while taking away food, cleaning or resting. The dataset presents several structured activities that are composed of different short-term actions, which are shared among different activities.

The Table $\Pi$ I shows the 6 different cooking recipes (activities) used in this dataset. 9 action classes are shown in bold font. In addition to training from these action classes, we train "undefined" action class using histogram features from the frames out of the ground-truth action boundaries. The dataset has 53 different long-term activities in total, where the first 28 activities are used for training. Each activity typically lasts between 3000-6000 frames and some activities may temporally overlap with each other. We define a "scene" as a various combination of these activities. We control the types of scenarios by selectively adding and removing the human objects. This allows us to have 93 different scenes for testing. Please refer to Fig. 4 for an example scenario of the dataset.

Figure 6 shows an example grammar we used for the activity Lemon sugar. (Refer to Table III for a full list of activity classes). The non-terminal symbols are defined in the same manner except for the noise symbol, which is added for robustness as similarly defined in [19], [54]. The noise symbol can expand to any terminal symbol, corresponding to the uniform probability on all symbols. It allows a parser to accept any symbol that are inconsistent with the defined activity structure. For other non-terminals except $S$ and $N$, the first row represents the probability of self-recursion. The second row is the symbol observation probability and the third row represents noise probability.

We test with two different scenarios in this experiment. The first scenario contains 50 different scenes

\footnotetext{
${ }^{1}$ The Crêpe dataset can be obtained on request by contacting the first author.
} 
TABLE II: Temporally structured cooking action descriptions of the proposed Crêpe dataset. Even though many actions are shared, there are characteristic actions and their orders that can distinguish each recipe from others.

\begin{tabular}{|l|l|}
\hline Activity Class & Action-based Description \\
\hline Lemon sugar & Stir/Pour/Spread mixture - Flip - Pour lemon juice - Sprinkle sugar - Fold \\
\hline Banana chocolate & Stir/Pour/Spread mixture - Cut banana - Flip - Transfer banana - Grate chocolate - Fold \\
\hline Cheese ham & Stir/Pour/Spread mixture - Cut ham - Grate cheese into bowl - Flip - Transfer cheese \& ham - Fold \\
\hline Cheese ham parsley & $\begin{array}{l}\text { Stir/Pour/Spread mixture - Cut ham - Grate cheese into bowl - Cut parsley - Flip - Transfer cheese\&ham - } \\
\text { Sprinkle parsley - Fold }\end{array}$ \\
\hline Goat cheese spinach & Stir/Pour/Spread mixture - Cut goat cheese - Flip - Transfer cheese and spinach - Fold \\
\hline $\begin{array}{l}\text { Goat cheese spinach } \\
\text { nutmeg }\end{array}$ & Stir/Pour/Spread mixture - Cut goat cheese - Flip - Transfer cheese\&spinach - Sprinkle nutmeg - Fold \\
\hline
\end{tabular}

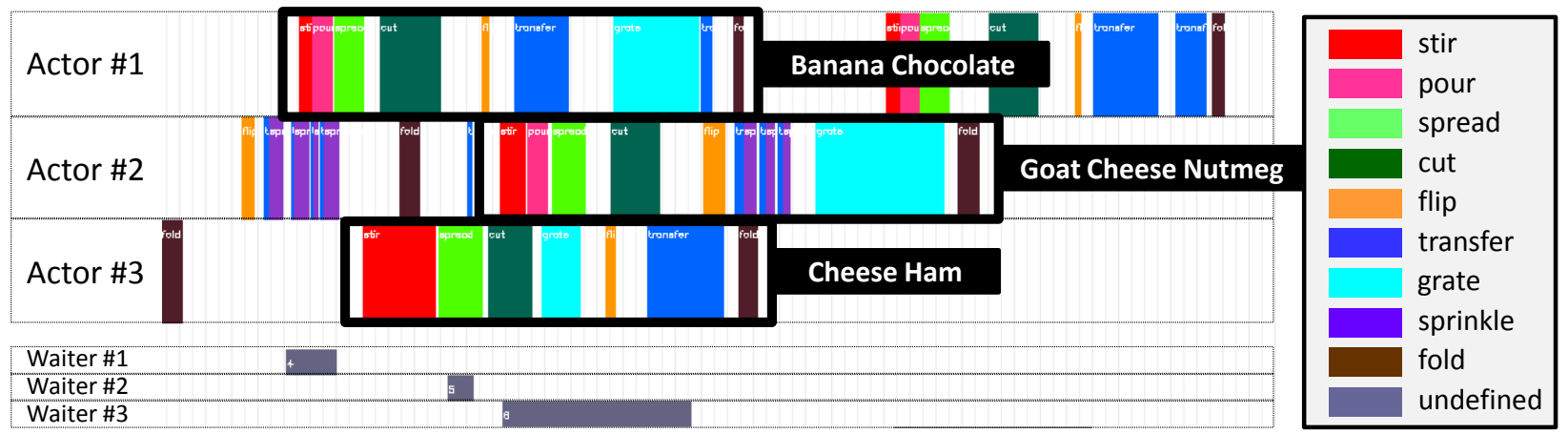

Fig. 4: An example scenario of the Crêpe dataset, where three chefs cook banana chocolate crêpe, goat cheese nutmeg crêpe and cheese ham crêpe, respectively. Three waiters are also in the background, who clean the table and bring plates to the chefs. The $\mathrm{x}$-axis represents time.

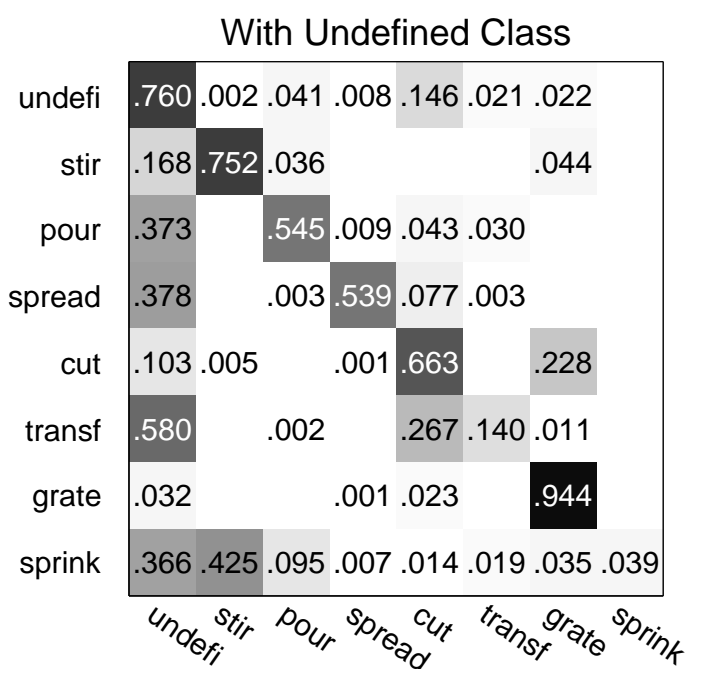

(a)

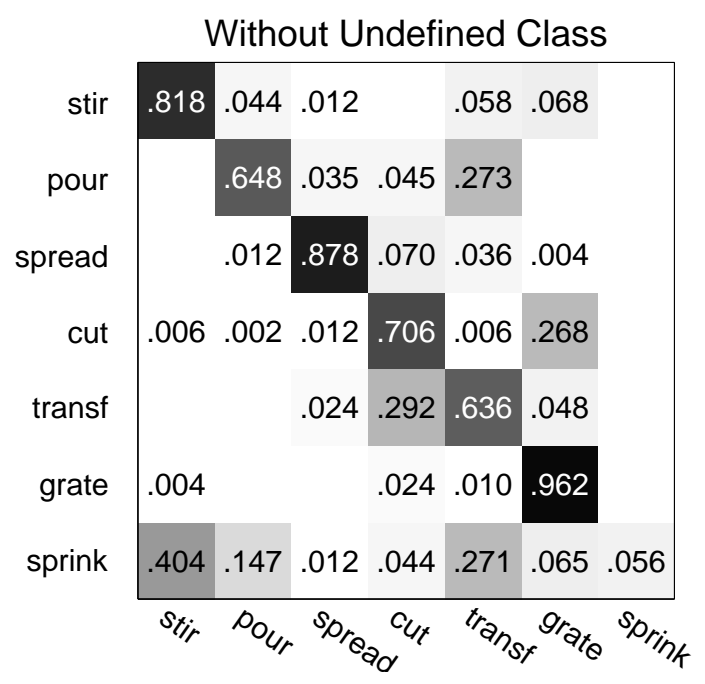

(b)

Fig. 5: Confusion matrices of recognized actions using the classification method described in Section IV-A (a) Including an undefined action, which is trained from randomly selected samples that do not belong to any of the defined classes. In our experiment, we include this undefined class. (b) The result with the actions defined in Table II only, for reference purpose. 


$\begin{array}{lllll}\mathrm{S} & \rightarrow & \mathrm{N} \text { I P D N F P R N } & \text { [1.0] } \\ \mathrm{N} & \rightarrow & \mathrm{c} & {[0.125]} \\ & \mid & \mathrm{p} & {[0.125]} \\ & \mid & \mathrm{i} & {[0.125]} \\ & \mid & \mathrm{d} & {[0.125]} \\ & & \mathrm{t} & {[0.125]} \\ & & \mathrm{r} & {[0.125]} \\ & \mid & \mathrm{f} & {[0.125]} \\ \mathrm{I} & \rightarrow & \mathrm{g} & {[0.125]} \\ & & \mathrm{I} & {[0.75]} \\ & \mid & \mathrm{N} & {[0.15]} \\ \mathrm{P} & \rightarrow & \mathrm{P} \mathrm{P} & {[0.10]} \\ & \mid & \mathrm{p} & {[0.75]} \\ & \mid & \mathrm{N} & {[0.15]}\end{array}$

(Other action non-terminals D, F, ... are defined similarly.)

Fig. 6: An example grammar for the activity Lemon sugar of Crêpe Dataset, which corresponds to the activity description defined in Table II

where at least two distractors and any number of chefs are included. The second scenario contains 34 scenes where at least two chefs and any number of distractors are included.

Figure 5 shows the action classification accuracies, implemented using the method described in Section IV-A Figure 5(a) shows the case where an undefined action, trained from randomly selected samples that do not belong to any of the defined classes, is included. We include this undefined class throughout our experiment. For reference purposes, we also include the result obtained using only the actions defined in Table II

The Figure $7 \mathrm{a}$ shows the scenario containing at least two chefs with any number of distractors. This is the case where multiple known activities need to be detected. The NMIA policy shows better performance than full sampling due to noise in action classification. On the other hand, the Figure $7 \mathrm{~b}$ shows a scenario containing at least two distractors with any number of chefs. This scenario contains actions that are not relevant to any specific activity, where attending at the correct information source (chefs) is critical.

It is important to note that in all of our policies except "Full sampling", we only attend one window at a time, which greatly reduces the amount of time required for low- and mid-level processes. As a trade off, it is expected that any system utilizing an attention policy will have a lower detection performance if there are many chefs (real activities). However, Figure 7a shows that this is not always the case as the NMIA system performs better than Full attention system based on Area Under Curve (AUC) scores (Full AUC $=0.577$ versus NMIA AUC=0.697).

It has been previously shown that a selective sampling does not necessarily deteriorate recognition/detection performance, e.g. [13], [42]. Sometimes it even improve the performance by filtering out noise [13], especially
TABLE III: AUC scores with additional noise conditions for a scenario having at least two chefs.

\begin{tabular}{|c|l|l|l|l|l|}
\hline Policy Noise & \multirow{2}{*}{$0 \%$} & $5 \%$ & $10 \%$ & $15 \%$ & $20 \%$ \\
\hline Full & 0.577 & 0.577 & 0.558 & 0.534 & 0.511 \\
\hline MEA & 0.598 & 0.598 & 0.589 & 0.577 & 0.516 \\
\hline MMIA & 0.589 & 0.589 & 0.589 & 0.554 & 0.516 \\
\hline NMIA & 0.697 & 0.697 & 0.682 & 0.612 & 0.525 \\
\hline
\end{tabular}

TABLE IV: AUC scores with additional noise conditions for a scenario having at least two distractors.

\begin{tabular}{|c|l|l|l|l|l|}
\hline Policy Noise & \multirow{2}{*}{$0 \%$} & $5 \%$ & $10 \%$ & $15 \%$ & $20 \%$ \\
\hline Full & 0.773 & 0.764 & 0.764 & 0.751 & 0.697 \\
\hline MEA & 0.634 & 0.625 & 0.625 & 0.610 & 0.577 \\
\hline MMIA & 0.677 & 0.672 & 0.672 & 0.663 & 0.623 \\
\hline NMIA & 0.682 & 0.667 & 0.667 & 0.658 & 0.623 \\
\hline
\end{tabular}

if the sampling strategy is well designed enough not to miss the valuable information of the problem. In [42], Figure 7 , the selective sampling does not miss critical points, resulting in a lower error rate compared to the uniform sampling. Furthermore, in [13], Tables 1 and 2 , the authors show that the recognition performance of selective sampling can be even higher than full sampling while maintaining shorter computational time especially when the action sequence is long and noisy.

A sample scenario is shown in Figure 8. The blue and red boxes show the currently attended window based on MMIA and MEA policies, respectively, among candidate windows. We show only MEA and MMIA policies to clearly demonstrate the comparison between these two contrasting policies. As explained in Section III-C, the MEA policy prefers a window with the lowest estimated score where MMIA policy prefers a window with the highest estimated score. In frame 5088, the chef in the middle (ID \#0) is finishing a previous task by folding the crêpe thus having a higher MEA and lower MMIA scores, while the chef on the left (ID \#1) is initiating a new task by pouring the mix, resulting in the opposite scores. In subsequent frames, ID \#7 and ID \#8 are waiter and waitress, respectively, who simply help chef by cleaning the table and bringing out the dishes. While MEA policy keeps focusing on ID \#1 from frame 6614, it can be observed that the MMIA policy actively jumps among 3 windows.

Finally, we test the robustness of our proposed attention policies by adding a noise perturbation to the symbol observation likelihoods. We run the experiments again with added noise from $5 \%$ to $20 \%$ with a step size of $5 \%$ to track the changes of AUC score. As can be seen in Tables III and IV the AUC scores do not change much until $10 \%$ noise perturbation and starts exhibiting some change at $15 \%$ mark. The cause of the small amount of changes would be due to the structural constraints defined in the grammars. However, it can be observed that there is a drastic change from the $20 \%$ mark. 


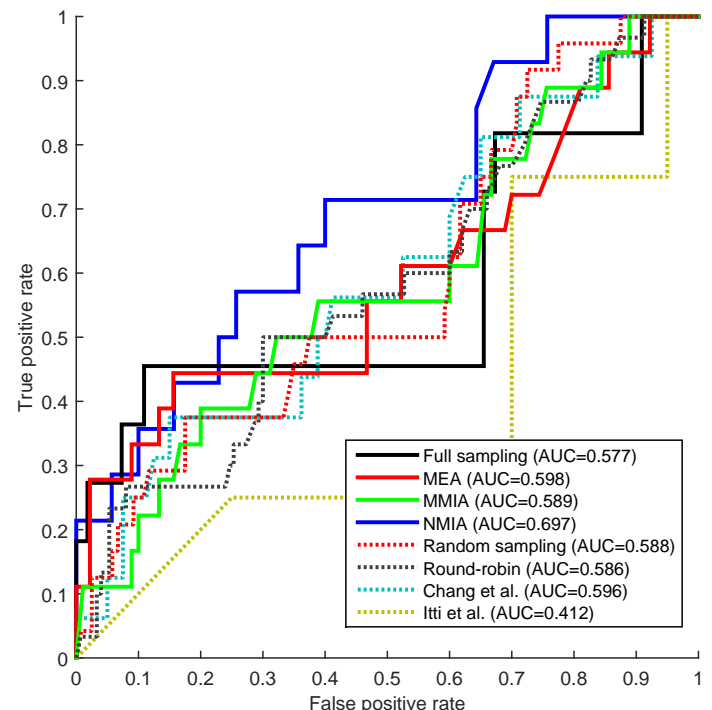

(a)

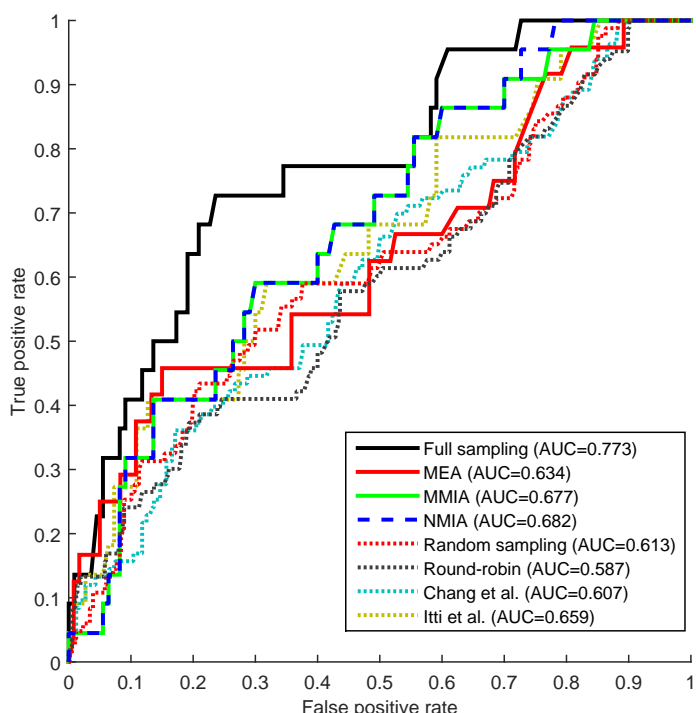

(b)

Fig. 7: (a) A scenario containing at least two chefs, which is the case where multiple known activities need to be detected. The attention systems generally show better performances than the Full sampling system. (b) A scenario containing at least two distractors, where the distractors perform actions that are irrelevant to cooking. The detection performance is better in overall as there is usually a clear difference in activity likelihoods of distractors than that of chefs. Best viewed in color.

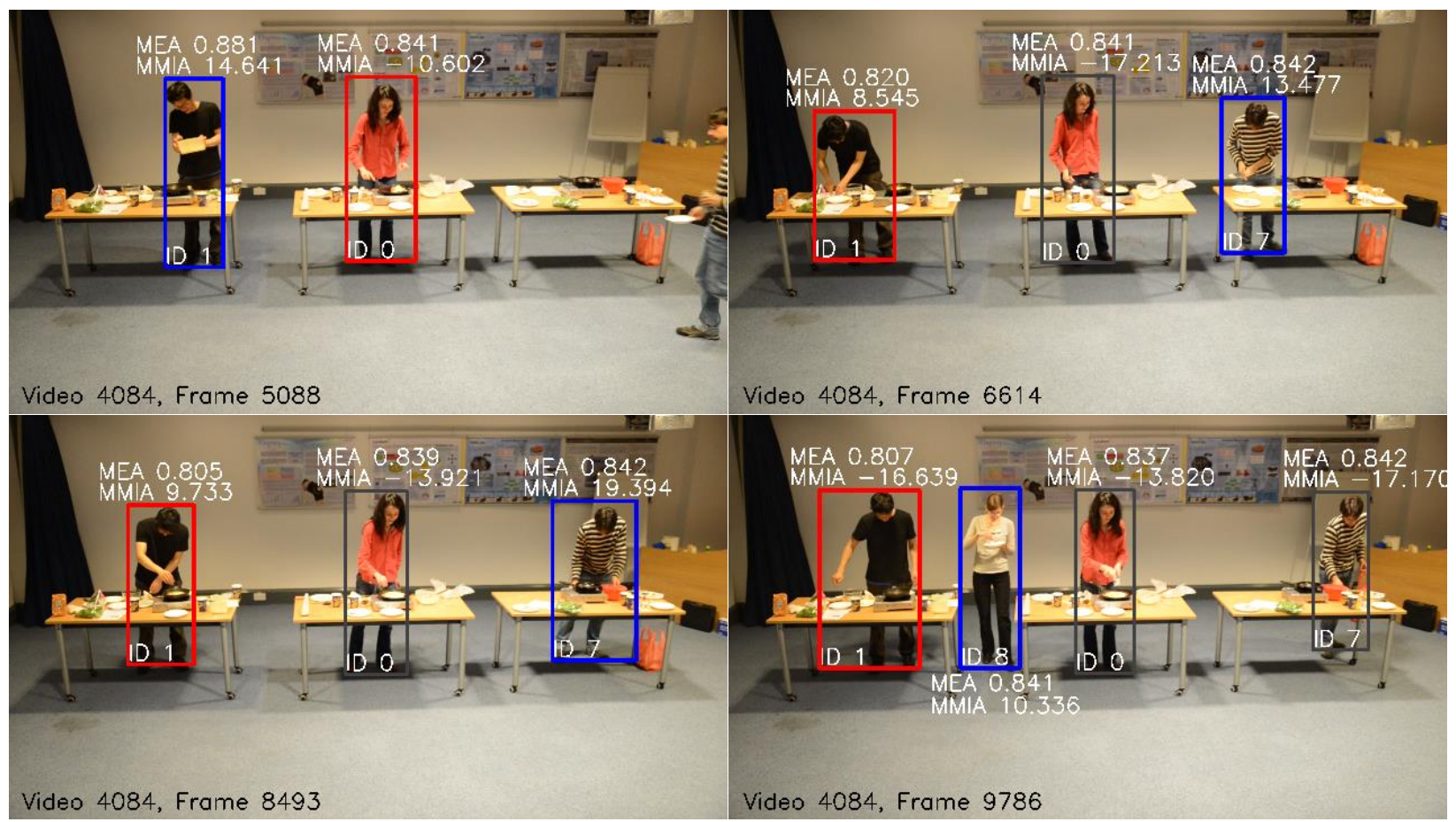

Fig. 8: A sample scenario. The blue and red box respectively shows the currently attended window based on MMIA and MEA policies among candidate windows. MEA policy chooses a window with the lowest score (lowest uncertainty) whereas MMIA policy chooses a window with tee highest score (highest information gain). In frame 5088, the chef in the middle (ID \#0) is finishing a previous task by folding the crêpe thus having a higher MEA and lower MMIA scores, while the chef on the left (ID \#1) is initiating a new task by pouring the mix, resulting in the opposite scores. In subsequent frames, ID \#7 and ID \#8 are distractors who simply helps chef by cleaning the table and bring out the dish. While MEA policy keeps focusing on \#1 from frame 6614, MMIA policy actively jumps among 3 windows. Best viewed in color. 


\section{Computational Cost}

We used an intel i7-2GHz, 12GB RAM, Ubuntu 12.10 laptop. The main code which implements various attention policies was programmed in Python, while the SCFG parser was programmed in $\mathrm{C}++$ compiled with GNU C++. To interface between the main code and the parser, we implemented a Python wrapper to directly access all the parser functionalities. The lowlevel feature extraction library [46] and the extremely clustered random forest [50] for generating codebook were both implemented in $\mathrm{C}++$. The random forest [51] we used for detecting actions were implemented in $\mathrm{C}++$ with Python bindings.

The majority of the time consumed while processing videos was in the low-level visual feature extraction part. The average processing time taken to compute a descriptor of a visual feature point was $20 \mathrm{~ms}$. On average, 75 descriptors were computed from each boundary window of a person per frame, resulting in 1.54 seconds on average per frame. The histogram computation and action classification using random forest was negligible.

A computational time overhead is shown in Figure 9. The different areas show the time spent for each process layer. $T_{\text {prediction }}$ is the overhead caused by using our predictive parsing method, $T_{\text {parsing }}$ is the parsing time required to compute the likelihood of an activity and $T_{\text {feature }}$ is the average time required to extract visual features per frame. Although the complexity of the SCFG parsing also increases over time due to the increased number of parsing hypotheses, we found that the amount of time required for parsing is reasonable because the parsing is performed every 2 seconds $(60$ frames) and the overall parsing and action prediction time was less than 1 second even after passing the 6000th frame on a consumer-grade computer.

It is worth mentioning that except for the Full attention method, all other attention methods attend only a single window at a time, resulting in nearly identical processing time of $T_{\text {prediction }}, T_{\text {parsing }}$ and $T_{\text {feature }}$ regardless of which sampling strategy used. If we assume that there are $N$ candidate windows on average in a given scene and the Full attention policy requires $T_{\text {feature }}$ for feature computation, then all other attention methods have the feature computation time of only $T_{\text {feature }} / N$.

\section{CONCLUSions \& Future Work}

We presented the Spatio-Temporal Attention Relocation (STARE) system, which is capable to dynamically allocate an attention to efficiently detect activities under resource-bounded condition. To realize our purpose, we presented an activity detection method based on SCFG capable of generating predictions of possible actions while recognizing input streams by considering the

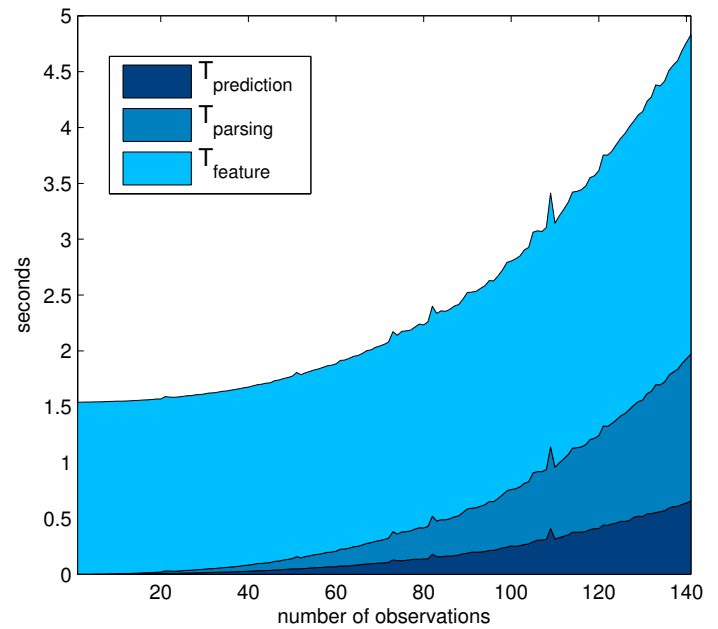

Fig. 9: Computational time overhead analysis. The different areas show the amount of time spent for each process layer. $T_{\text {prediction }}$ is the overhead caused by using our predictive parsing method, $T_{\text {parsing }}$ is the parsing time required to compute the likelihood of an activity and $T_{\text {feature }}$ is the average time required to extract spatio-temporal features per frame. The sampling interval between observations is 60 .

structural information of activities. We also proposed three attention policies calculated from the amount of information contained in an action. Each of the policies showed a characteristic performance for different scene situations. For evaluation, we presented a new structured activity dataset of concurrent multiple human objects of high resolution video. Through the whole evaluation the STARE achieved the comparable activity detection performance while consuming a relatively much lower computational load.

As shown in Section IV-D, the additional computational complexity required for computing an attention policy is reasonable. Although the system has been only tested in offline due to the high complexity in low-level processing, it would not be impossible to run in near-real time by adopting parallel processing paradigm in both visual feature extraction and SCFG parsing. As another extension, we plan to study a unified framework of the three attentional policies which can dynamically change the best policy depending on the scenario progression. Also, instead of attending only a single window at a time, the number of windows the system can attend at a time can be changed depending on the amount of resources available. Furthermore, a bottom-up attention approach can be combined together to improve the detection performance by incorporating low-level saliency features and spatial predictions.

\section{ACKNOWLEDGMENT}

We acknowledge Miguel Sarabia del Castillo for improving the efficiency of the original SCFG parser used in [19]. This work was 
partially funded by the European Commission Seventh Framework Programme projects DARWIN (FP7-270138) and WYSIWYD (FP7612139). The Crêpe dataset collection was funded by EPSRC Network on Vision and Language, under grant scheme Pump-Priming V\&L Research 2013-1.

\section{REFERENCES}

[1] A. Andreopoulos and J. K. Tsotsos, "A theory of active object localization," in ICCV, 2009, pp. 903-910.

[2] A. Borji and L. Itti, "State-of-the-art in visual attention modeling," IEEE TPAMI, vol. 35, no. 1, pp. 185-207, 2013.

[3] A. Davison and D. Murray, "Simultaneous localization and mapbuilding using active vision," IEEE TPAMI, pp. 865-880, 2002.

[4] H. Larochelle and G. E. Hinton, "Learning to combine foveal glimpses with a third-order boltzmann machine," in NIPS, 2010, pp. 1243-1251.

[5] J. Denzler and C. Brown, "Information theoretic sensor data selection for active object recognition and state estimation," IEEE TPAMI, vol. 24 , no. 2, pp. 145-157, 2002

[6] E. Sommerlade and I. Reid, "Probabilistic surveillance with multiple active cameras," in IEEE International Conference on Robotics and Automation, 2010, pp. 440-445.

[7] C. Micheloni, B. Rinner, and G. L. Foresti, "Video analysis in pan-tiltzoom camera networks," Signal Processing Magazine, IEEE, vol. 27, no. 5, pp. 78-90, 2010.

[8] P. Jiang et al., "Salient region detection by ufo: uniqueness, focusness and objectness," in ICCV, 2013.

[9] S. Vijayanarasimhan and A. Kapoor, "Visual recognition and detection under bounded computational resources," CVPR, pp. 1006-1013, 2010.

[10] D. Ognibene and G. Baldassarre, "Ecological active vision: four bioinspired principles to integrate bottom-up and adaptive top-down attention tested with a simple camera-arm robot," IEEE Transactions on Autonomous Mental Development, pp. 1-24, 2014.

[11] L. Paletta, G. Fritz, and C. Seifert, "Cascaded sequential attention for object recognition with informative local descriptors and q-learning of grouping strategies," in CVPR Workshope, Los Alamitos, CA, USA: IEEE, 2005, p. 94

[12] N. Oliver and E. Horvitz, "Selective perception policies for guiding sensing and computation in multimodal systems: a comparative analysis," Computer Vision and Image Understanding, vol. 100, no. 1 pp. 198-224, 2005.

[13] H. J. Chang et al., "Action chart: a representation for efficient recognition of complex activities," in BMVC, 2013

[14] D. Ognibene and Y. Demiris, "Towards active events recognition," in International Joint Conference on Artificila Intelligence, 2013.

[15] A. Oikonomopoulos, I. Patras, and M. Pantic, "Spatiotemporal localization and categorization of human actions in unsegmented image sequences," IEEE Trans Im. Proc., vol. 20, no. 4, pp. 1126-1140, Apr 2011 .

[16] X. Chang, W.-S. Zheng, and J. Zhang, "Learning person-person interaction in collective activity recognition," IEEE Trans. on Im. Proc., vol 24, no. 6, pp. 1905-1918, Jun. 2015.

[17] J. Aggarwal and M. Ryoo, "Human activity analysis: a review," ACM Comput. Surv., vol. 43, no. 3, p. 16, 2011

[18] M. Ryoo and J. Aggarwal, "Stochastic representation and recognition of high-level group activities," International Journal of Computer Vision, pp. 1-18, 2010.

[19] Y. Ivanov and A. Bobick, "Recognition of visual activities and interactions by stochastic parsing," IEEE TPAMI, vol. 22, pp. 852-872, 2000

[20] K. Lee et al., "A syntactic approach to robot imitation learning using probabilistic activity grammars," Rob. Auton. Syst., vol. 61, no. 12, pp. 1323-1334, 2013.

[21] D. Moore and I. Essa, "Recognizing multitasked activities from video using stochastic context-free grammar," in Proceedings of the National Conference on Artificial Intelligence, Menlo Park, CA; Cambridge, MA London; AAAI Press; MIT Press; 1999, 2002, pp. 770-776.

[22] D. Minnen, I. Essa, and T. Starner, "Expectation grammars: leveraging high-level expectations for activity recognition," CVPR, 2003.

[23] K. Kitani, S. Yoichi, and A. Sugimoto, "Recovering the basic structure of human activities from noisy video-based symbol strings," Int. J. Pattern Recognit Artif Intell., vol. 22, no. 08, pp. 1621-1646, 2008.

[24] K. Lee and Y. Demiris, "Towards incremental learning of taskdependent action sequences using probabilistic parsing," in IEEE International Conference on Development and Learning, vol. 2, Frankfurt, Germany, 2011, pp. 1-6.

[25] Y. J. Lee, J. Ghosh, and K. Grauman, "Discovering important people and objects for egocentric video summarization," in CVPR, IEEE, 2012 pp. $1346-1353$

[26] K. Lee, T. K. Kim, and Y. Demiris, "Learning action symbols for hierarchical grammar induction," in ICPR, Tsukuba Science City, Japan, 2012, pp. 3778-3782.

[27] K. Lee, T. K. Kim, and Y. Demiris, "Learning reusable task components using hierarchical activity grammars with uncertainties," in IEEE International Conference on Robotics and Automation, St. Paul, USA,

[28] K. Friston and S. Kiebel, "Predictive coding under the free-energy principle," Philosophical Transactions of the Royal Society B: Biological Sciences, vol. 364, no. 1521, p. 1211, 2009.
[29] D. Ognibene et al., "Hierarchies for embodied action perception," English, in Computational and Robotic Models of the Hierarchical Organization of Behavior, G. Baldassarre and M. Mirolli, Eds., Springer Berlin Heidelberg, 2013, pp. 81-98.

[30] L. Zhang, Z. Zeng, and Q. Ji, "Probabilistic image modeling with an extended chain graph for human activity recognition and image segmentation," IEEE TPAMI, vol. 20, no. 9, pp. 2401-2413, Sep. 2011.

[31] T. S. Lee and X. Y. Stella, "An information-theoretic framework for understanding saccadic eye movements.", in NIPS, 1999, pp. 834-840.

[32] G. Rotman et al., "Eye movements when observing predictable and unpredictable actions," Journal of Neurophysiology, vol. 96, no. 3, p. $1358,2006$.

[33] D. Ognibene et al., "Contextual action recognition and target localization with an active allocation of attention on a humanoid robot," Bioinspiration \& biomimetics, vol. 8, no. 3, p. 035002, 2013.

[34] K. Friston et al., "Perceptions as hypotheses: saccades as experiments," Frontiers in Psychology, vol. 3, no. 151, pp. 151-170, 2012.

[35] K. Friston et al., "Active inference and epistemic value," Cogn. Neuroscie., pp. 1-28, 2015, PMID: 25689102.

[36] B. W. Tatler et al., "Eye guidance in natural vision: reinterpreting salience," J. Vis., vol. 11, no. 5, pp. 1-23, 2011.

[37] J. Gottlieb et al., "Information-seeking, curiosity, and attention: computational and neural mechanisms," Trends in cognitive sciences, vol. 17 , no. 11 , pp. 585-593, 2013.

[38] L. Itti, C. Koch, and E. Niebur, "A model of saliency-based visual attention for rapid scene analysis," IEEE TPAMI, vol. 20, no. 11, pp. 1254-1259, 1998 .

[39] L. Itti and P. Baldi, "A principled approach to detecting surprising events in video," in CVPR, vol. 1, 2005, pp. 631-637.

[40] L. Itti, N. Dhavale, and F. Pighin, "Realistic avatar eye and head animation using a neurobiological model of visual attention," in Proc. 48th SPIE Int. Symp., B. Bosacchi, D. B. Fogel, and J. C. Bezdek, Eds., vol. 5200, Bellingham, WA: SPIE Press, Aug. 2003, pp. 64-78.

[41] J. Denzler, M. Zobel, and H. Niemann, "Information theoretic focal length selection for real-time active $3 \mathrm{~d}$ object tracking," in ICCV, 2003, pp. $400-407$

[42] H. J. Chang, H. Jeong, and J. Y. Choi, "Active attentional sampling for speed-up of background subtraction," in CVPR, IEEE, 2012, pp. 20882095.

[43] V. Navalpakkam and L. Itti, "Modeling the influence of task on attention," Vision Res., vol. 45, no. 2, pp. 205-231, 2005.

[44] M. Ryoo and W. Yu, "One video is sufficient? human activity recognition using active video composition," WMVC, 2011.

[45] M. Hoai and F. De la Torre, "Max-margin early event detectors," in CVPR, 2012

[46] H. Wang et al., "Action recognition by dense trajectories," in CVPR, 2011.

[47] N. Dalal, B. Triggs, and C. Schmid, "Human detection using oriented histograms of flow and appearance," ECCV, pp. 428-441, 2006.

[48] I. Laptev et al., "Learning realistic human actions from movies," in CVPR, 2008, pp. 1-8.

[49] G. Yu, J. Yuan, and Z. Liu, "Action search by example using randomized visual vocabularies," IEEE Trans Im. Proc., vol. 22, no. 1, pp. 377-390, Jan. 2013.

[50] F. Moosmann, B. Triggs, F. Jurie, et al. "Fast discriminative visual codebooks using randomized clustering forests," NIPS, pp. 985-992, 2007.

[51] F. Pedregosa et al., "Scikit-learn: machine learning in Python," Journal of Machine Learning Research, vol. 12, pp. 2825-2830, 2011.

[52] H. Wang et al., "Action recognition using nonnegative action component representation and sparse basis selection," IEEE Trans Im. Proc., vol. 23 , no. 2, pp. 570-581, Feb. 2014

[53] K. Lee, "A syntactic approach to robot learning of human tasks from demonstrations," $\mathrm{PhD}$ thesis, Imperial College London, 2013.

[54] A. F. Bobick and Y. A. Ivanov, "Action recognition using probabilistic parsing," in CVPR, 1998, pp. 196-202.

[55] A. Stolcke, "An efficient probabilistic context-free parsing algorithm that computes prefix probabilities," in Computational Linguistics, vol. 21, MIT Press, 1995.

[56] A. Kraskov et al., "Hierarchical clustering using mutual information," EPL (Europhysics Letters), vol. 70, no. 2, p. 278, 2005.

[57] N. X. Vinh, J. Epps, and J. Bailey, "Information theoretic measures for clusterings comparison: variants, properties, normalization and correction for chance," The Journal of Machine Learning Research, vol. 11, pp. 2837-2854, 2010.

[58] A. Ogale, A. Karapurkar, and Y. Aloimonos, "View-invariant modeling and recognition of human actions using grammars," Dynamical Vision, pp. 115-126, 2007.

[59] P. Langley and S. Stromsten, "Learning context-free grammars with a simplicity bias," in The European Conference on Machine Learning, vol. 1810,2000 , pp. $220-228$.

[60] A. Stolcke and S. Omohundro, "Inducing probabilistic grammars by bayesian model merging," Grammatical Inference and Applications, vol. 862, pp. 106-118, 1994.

[61] S. Oh et al., "A large-scale benchmark dataset for event recognition in surveillance video," in CVPR, 2011, pp. 3153-3160. 